\title{
Immunomodulatory Effect of CpG ODN-Adjuvanted Bacterin against Salmonella enterica serovar Enteritidis in Broiler Chickens
}

\author{
Mohamed Abed ${ }^{1}$, Mahmoud Elhariri ${ }^{2}$, Rehab El-Helw ${ }^{2}$, Marwa S. Khattab ${ }^{3}$, Ahmed Setta $^{4}$ and Rafik Soliman $^{2}$ \\ ${ }^{1}$ Ceva Sante Animale, Cairo, Egypt \\ ${ }^{2}$ Department of Microbiology, Faculty of Veterinary Medicine, Cairo University, Giza, 12211, Egypt \\ ${ }^{3}$ Department of Pathology, Faculty of Veterinary Medicine, Cairo University, Giza, 12211, Egypt \\ ${ }^{4}$ Department of Poultry Diseases, Faculty of Veterinary Medicine, Cairo University, Giza, 12211, Egypt \\ *Corresponding author's Email: ahmed.setta@cu.edu.eg; (DorciD: 0000-0002-9088-6233
}

\begin{abstract}
Bacterial oligodeoxynucleotide containing Cytosine Guanine motifs (CpG-ODN) has been reported to induce immunostimulatory activity against a variety of bacterial, viral, and protozoan infections in a wide range of vertebrate species. The objective of this study was to investigate the dose-dependent immunomodulatory effect of CpG ODN on Salmonella Enteritidis bacterin in broiler chickens. Two hundred one-day-old broiler chicks, divided into 5 groups, were used in this study. First three groups were immunized with Salmonella Enteritidis bacterin adjuvanted with different doses of $\mathrm{CpG}$ ODN $(50 \mu \mathrm{g}, 100 \mu \mathrm{g}$ and $200 \mu \mathrm{g})$. The control groups included a group that was immunized with Salmonella Enteritidis bacterin adjuvanted with aluminum hydroxide and a non-immunized group. The intestinal colonization, cellular responses, mucosal and systemic immune responses of immunized chickens was measured at different intervals, until 42 days of age. At two weeks post-immunization, 20 chicks from each group were orally challenged by Salmonella Enteritidis fresh bacterial culture $\left(1.2 \times 10^{8} \mathrm{CFU} / \mathrm{ml}\right)$. The survival rates and the pathological changes of challenged chickens in the different groups were monitored for extra 10 days. Compared to the aluminum hydroxide adjuvanted bacterin, the $\mathrm{CpG}-\mathrm{ODN}$ adjuvant bacterin induced significant protection and improved survival rate of challenged chickens. Also Salmonella Enteritidis was not recovered from the intestinal tract of vaccinated challenged groups. There was a significant dose-dependent immunostimulatory adjuvant effect of CPG-ODN on the level of secretory IgA and the induced mucosal responses. The 200-CpG ODN group showed the highest IgA response followed by $100-\mathrm{CpG}$ ODN group then the 50-CpG ODN and the aluminum hydroxide groups $(\mathrm{P}<0.05)$. Also, cellular interactions were remarkably reduced in the liver and intestine of $\mathrm{CpG}$ ODN-treated chickens. No inflammatory cellular infiltrations were seen in the liver and intestine of 200-CpG ODN treated group. In conclusion, the presented findings have shown the significant immunostimulatory effect of CpGODN and its effect on Salmonella Enteritidis bacterin in controlling Salmonella infection in broiler chickens.
\end{abstract}

Keywords: Cellular responses, CpG ODN, Mucosal immunity, Salmonella Enteritidis

\section{INTRODUCTION}

Salmonella enterica subspecies enterica is a leading cause of global food-borne zoonosis. Poultry and poultry-derived food remain the main source of infection with non-host adapted serovars, including Salmonella Enteritidis and Salmonella Typhimurium (Burr et al., 2005; Much et al., 2007; Stephens et al., 2007), hence they are good colonizers of the chicken caeca without inducing apparent clinical manifestations (Barrow, 2007). Controlling such kind of silent infection to reduce potential human transmission remains of global interest.

Host-pathogen interaction is a dynamic process which modulates the host immune response to infection (Janeway and Medzhitov, 2002). The host innate immune system recognizes pathogens via a wide range of recognition components called pathogen recognition receptors (PRRs) which recognize pathogen conserved motifs called pathogen associated molecular patterns (PAMPs) that are expressed by the invading infectious microbes, including pathogen cellsurface components, such as bacterial LPS and or pathogen nucleic acid, including single- and double-stranded RNA and CpG DNA (Janeway and Medzhitov, 2002).

Bacterial DNA is a potent stimulator of innate immune responses which is reasoned by the presence of unmethylated $\mathrm{CpG}$ dinucleotide (Krieg, 2002). Indeed, it has been shown that bacterial DNA and synthetic oligodeoxynucleotide containing unmethylated $\mathrm{CpG}$-dinucleotides (CpG-ODN) stimulate host responses during infections. Previous literatures have demonstrated that bacterial DNA and CpG-ODN components induce nitric oxide and oxygen production and intracellular killing of Salmonella in avian macrophages and heterophils (Xie et al., 2003; He et al., 2007). In mammals, the CpG-ODN motifs are recognized by Toll-like receptor (TLR-9) leading to stimulation of several types of innate and acquired immune cells, including phagocytes, lymphocytes and polymorph nuclear cells 
(Ribeiro and Schijns, 2010; Tovey and Lallemand, 2010). This stimulation leads to activation of Th-1 cellular immune responses via induction of proinflammatory cytokines and chemokines, including interleukins (IL-1, IL-6, IL-18) and interferon (IFN- $\delta$ ) (Ribeiro and Schijns, 2010). Unlike mammals, chickens respond to CpG-ODN through TLR-21 and TLR-15 (Keestra et al., 2010; Ciraci and Lamont, 2011). The present study investigated the dose-dependent effect of $\mathrm{CpG}-\mathrm{ODN}$ as a vaccine candidate and its effect on intestinal colonization, local and humoral immune responses and cellular interactions in chickens in response to infection with Salmonella Enteritidis.

\section{MATERIALS AND METHODS}

\section{Bacterial strain}

Field isolates of Salmonella Enteritidis recovered from 25-day old broilers with, Diarrhea, cloacitis, hepatitis, and splenitis were obtained from the Biotechnology Centre for Research \& Services (CBRS), Faculty of Veterinary Medicine, Cairo University, Egypt. Aliquots of the bacteria were stored at $-70^{\circ} \mathrm{C}$ in a $50 \%$ brain heart infusion broth (BHI; Oxoid) supplemented with $25 \%$ (w/v) glycerol. This bacterial strain was fully identified using bacteriological and molecular methods.

\section{Preparation of Salmonella Enteritidis bacterial cultures}

Two to three colonies of Salmonella Enteritidis bacteria were added to $200 \mathrm{ml}$ of Luria Broth (LB) in a $1000 \mathrm{ml}$ Erlenmeyer flask. To obtain Salmonella Enteritidis in the stationary phase, the cultures were grown at $37^{\circ} \mathrm{C}$ for $16-18$ hours with shaking at $200 \mathrm{rpm} / \mathrm{min}$. The stationary phase cultures contained approximately $1 \times 10^{9} \mathrm{CFU} / \mathrm{ml}$ of Salmonella Enteritidis was used for the preparation of bacterin. Preparation Salmonella Enteritidis challenge culture include growing of Salmonella Enteritidis in the logarithmic growth phase where a 1:1000 dilution of the stationary phase culture was added to $100 \mathrm{ml}$ of Luria Broth in a 500-ml Erlenmeyer flask and incubated at 37oC for three hours with shaking at 200 $\mathrm{rpm}$. After shaking, the culture contained approximately $1 \times 10^{9} \mathrm{CFU}$ per ml. This culture was further diluted with saline to the concentration of bacteria required for the challenge experiments. Serial dilutions were plated in duplicate on Tryptose soy agar plates and incubated for $18-24$ hours at $37^{\circ} \mathrm{C}$. Following incubation, bacterial colonies on the plates were counted to validate the challenge dose.

\section{Ethical approval}

All procedures involving animals were done according to a protocol approved by the Faculty of Veterinary Medicine, Cairo University Committee on Animal Care and Ethics in accordance with research regulations. All dead chickens were humanly handled.

\section{Animal model development}

Chickens were randomly allocated to five groups (forty chicks per group) and housed. All groups were given free access to water and commercial starter broiler rations. The photoperiod was set at 24 hours daily for the first three days and 16 hours per day for the remaining seven days. Room temperature was maintained at $30-32{ }^{\circ} \mathrm{C}$ for the first week and 28-30 ${ }^{\circ} \mathrm{C}$ for the second week.

\section{Experimental birds}

A total of 200 one-day-old chickens (Cobb 500) were kept in closed pens at a maximum initial density of 15 birds per $\mathrm{m}^{2}$ according to the breed company instructions. Wood shavings were used as litter. The birds had access to commercial diet and water ad libitum. Ambient temperature and ventilation were regulated in keeping with standard breeding practices.

\section{Experimental design and Salmonella infection}

Two hundred chickens were randomly distributed into five groups $(n=40)$. At the second day of age, chickens in the first four groups were immunized with S. Enteritidis bacterin. The chicks in group one were immunized with S. Enteritidis bacterin adjuvanted with Aluminium hydroxide (AHG). Chickens in group two were immunized with S. Enteritidis bacterin adjuvanted with $50 \mu \mathrm{g}$ ODN-DNA (50-ODNG). Chickens in group three were immunized with S. Enteritidis bacterin adjuvanted with100 $\mu \mathrm{g}$ ODN-DNA (100-ODNG) and chickens in groups four were immunized with S. Enteritidis bacterin adjuvanted with $200 \mu \mathrm{g}$ ODN-DNA (200-ODNG). Chickens in group five were injected with phosphate buffered saline (PBS) and kept as negative control group (NCG). Two weeks post immunization 20 chicks from each group were challenged orally with $100 \mu \mathrm{l}$ of Salmonella Enteritidis strain containing $1.2 \times 10^{8} \mathrm{CFU} / \mathrm{ml}$ of stationary phase culture of Salmonella Enteritidis. From the remaining chickens in all groups sera and intestinal samples were collected at 18, 35 and 42 days of age for determination of humoral and mucosal immune responses. 
Effect of different adjuvants in Salmonella Enteritidis bacterin on the level of intestinal secretory immunoglobulin A (SIgA)

The concentration of SIgA was evaluated in all experimented groups at 18, 35 and 42 days of age by sandwich enzyme-linked immunosorbent assay (NOVA, Beijing, China).

Measurement of Salmonella Enteritidis-specific immunoglobulins titer in the immunized chickens

Blood samples were collected from 3 chickens/group from all 5 groups. Serum was separated and stored at $-80^{\circ} \mathrm{C}$ till examined. Salmonella Enteritidis-specific immunoglobulins were determined using a commercial ELISA kit (IDEXX Salmonella Enteritidis Ab X2 Test Kit; IDEXX Laboratories, Westbrook, Maine, USA). Serum samples with S/P ratios of less or equal to 0.2 should be considered negative. Samples with S/P ratios greater than 0.2 was considered as positive.

\section{Histopathology}

Liver and intestinal tissue samples were collected at 35 days of age from the chickens (three chickens per group) representing all treated chicken groups examined at necropsy were fixed in $10 \%$ neutral buffered formalin, trimmed, routinely processed, paraffin embedded, sectioned at $4 \mathrm{~mm}$ thickness, and stained with hematoxylin and eosin (H\&E) for histopathological examination (Setta et al., 2012a).

\section{Statistical analysis}

Data were analysed using one-way ANOVA test using Graph Pad software. Differences between groups were considered significant if $\mathrm{P}<0.05$.

\section{RESULTS}

Effect of immunization with CpG ODN adjuvanted Salmonella Enteritidis bacterin on survival rate of Salmonella Enteritidis challenged chickens

The survival rate of challenged chickens was recorded in differently treated chicks for 10 days following infection with Salmonella Enteritidis (Table 1). The CpG-ODN adjuvanted Salmonella Enteritidis bacterin had significantly improved the survival rate of Salmonella Enteritidis-infected chickens (75-85\%) when compared to aluminum hydroxide adjuvanted Salmonella Enteritidis bacterin control group (65\%). Interestingly, the survival rate of birds immunized with Salmonella Enteritidis bacterin adjuvanted with $200 \mu \mathrm{g}$ of $\mathrm{CpG}-\mathrm{ODN}$ induced higher survival rate (85\%) compared to the other tested groups.

Effect of CpG-ODN adjuvanted Salmonella Enteritidis bacterin on intestinal colonization of Salmonella Enteritidis in immunized chickens

Table 2 presents the rates of recovery of Salmonella Enteritidis from vaccinated challenged chickens groups. Triplicate samples were taken from chickens of each group at 42 day of age. Chicken groups immunized with CpG-ODN adjuvanted Salmonella Enteritidis bacterin were able to clear Salmonella Enteritidis colonization from the gut. Salmonella Enteritidis bacteria were not detected in all tested groups. In the control group, however, only three positive samples by $4.27 \log _{10}$ colony forming unit/gram (CFU/g) were recorded.

Mucosal immune response and SIgA production in chicken immunized by differently adjuvanted Salmonella Enteritidis bacterin

The concentration of SIgA was measured using ELISA in all experimented chicken groups at 18, 35 and 42 days of age. The results presented in table 3 showed that the highest SIgA concentration was recorded in chicken groups which immunized Salmonella Enteritidis bacterin adjuvanted with CpG ODN and aluminum hydroxide (AHG) as compared with the non-immunized control group (NCG). A dose dependent effect of CpG ODN on induction of SIgA was recorded. In comparison with the aluminum hydroxide adjuvant, the $\mathrm{CpG}$ ODN appeared to have strong immune stimulatory activity on SIgA response in all tested time points. The 200-ODNG group showed the highest response followed by $100-\mathrm{ODNG}$ group then the $50-\mathrm{ODNG}$ and the AHG groups $(\mathrm{P}<0.05)$.

Effect of adjuvant type and concentration on the humoral immune responses to Salmonella Enteritidis bacterin

Salmonella Enteritidis-specific antibodies in blood of differently immunized chickens were measured using ELISA (Table 4). No statistical differences were seen between the different groups although numerical differences were observed in the tested groups compared to the non-vaccinated controls. The CpG-ODN appeared to have strong immune stimulatory activity on Salmonella-specific antibodies response in comparison with AHG in all tested ages. The 200ODNG group showed the best humoral immune response followed by the 100-ODNG group then finally 50-ODNG and AHG group. 


\section{Cellular responses following Salmonella Enteritidis infection in immunized chickens}

Avian-Salmonella interactions were reported at the cellular level in intestinal and liver tissues at 35 days of age using $\mathrm{H} \& \mathrm{E}$ histopathological staining (Figure 1). Comparable to serological results, histopathogical examination of liver and intestinal tissue samples taken from chickens in the 200-ODNG groups showed no marked cellular changes. Nonetheless, tissue samples from AHG have shown mild to moderate tissue reaction, including inflammatory mononuclear cell infiltration as a result of Salmonella interaction at the cellular level.

Table 1. Effect of $\mathrm{CpG}$ adjuvant in Salmonella Enteritidis bacterin on the survival rate in different groups of broiler chickens challenged with Salmonella Enteritidis

\begin{tabular}{|c|c|c|c|c|c|c|c|c|c|c|}
\hline \multirow{3}{*}{$\begin{array}{l}\text { Days post- } \\
\text { challenge }\end{array}$} & \multirow{2}{*}{\multicolumn{2}{|c|}{$\begin{array}{c}\text { Non } \\
\text { Vaccinated } \\
\text { group }\end{array}$}} & \multicolumn{8}{|c|}{ Groups vaccinated with Salmonella Enteritidis bacterin adjuvanted with } \\
\hline & & & \multicolumn{2}{|c|}{ AHG } & \multicolumn{2}{|c|}{ 50-ODNG } & \multicolumn{2}{|c|}{ 100-ODNG } & \multicolumn{2}{|c|}{ 200-ODNG } \\
\hline & No. & $\%$ & No. & $\%$ & No. & $\%$ & No. & $\%$ & No. & $\%$ \\
\hline $1^{\text {st }}$ & $19 / 20$ & 95 & $19 / 20$ & 95 & $17 / 20$ & 85 & $19 / 20$ & 95 & $19 / 20$ & 95 \\
\hline $2^{\text {nd }}$ & $19 / 20$ & 95 & $19 / 20$ & 95 & $17 / 20$ & 85 & $19 / 20$ & 95 & $19 / 20$ & 95 \\
\hline $3^{\text {rd }}$ & $17 / 20$ & 85 & $19 / 20$ & 95 & $17 / 20$ & 85 & $19 / 20$ & 95 & $19 / 20$ & 95 \\
\hline $4^{\text {th }}$ & $17 / 20$ & 85 & $17 / 20$ & 85 & $17 / 20$ & 85 & $19 / 20$ & 95 & $19 / 20$ & 95 \\
\hline $5^{\text {th }}$ & $15 / 20$ & 75 & $17 / 20$ & 85 & $17 / 20$ & 85 & $19 / 20$ & 95 & $19 / 20$ & 95 \\
\hline $6^{\text {th }}$ & $14 / 20$ & 70 & $17 / 20$ & 85 & $17 / 20$ & 85 & $17 / 20$ & 85 & $19 / 20$ & 95 \\
\hline $7^{\text {th }}$ & $13 / 20$ & 65 & $15 / 20$ & 75 & $15 / 20$ & 75 & $17 / 20$ & 85 & $19 / 20$ & 95 \\
\hline $8^{\text {th }}$ & $13 / 20$ & 65 & $15 / 20$ & 75 & $15 / 20$ & 75 & $17 / 20$ & 85 & $19 / 20$ & 95 \\
\hline $9^{\text {th }}$ & $13 / 20$ & 65 & $15 / 20$ & 75 & $15 / 20$ & 75 & $17 / 20$ & 85 & $17 / 20$ & 85 \\
\hline $10^{\text {th }}$ & $13 / 20$ & 65 & $15 / 20$ & 75 & $15 / 20$ & 75 & $14 / 20$ & 70 & $17 / 20$ & 85 \\
\hline
\end{tabular}

AHG: Aluminium hydroxide group, 50-ODNG: $50 \mu \mathrm{g}$ ODN-DNA group, 100-ODNG: $100 \mu \mathrm{g}$ ODN-DNA group, 200-ODNG: $200 \mu \mathrm{g}$ ODN-DNA group.

Table 2. Effect of CpG-ODN adjuvant in Salmonella Enteritidis bacterin on the intestinal colonization of Salmonella Enteritidis in broiler chickens of different groups

\begin{tabular}{|c|c|c|c|}
\hline $\begin{array}{l}\text { Chicken Groups Immunized with } S \text {. Enteritidis } \\
\text { Bacterin adjuvanted with: }\end{array}$ & $\begin{array}{c}\text { No. of } \\
\text { Samples }\end{array}$ & $\begin{array}{c}\text { Salmonella } \\
\text { positive }\end{array}$ & Log CFU/g \\
\hline $\mathrm{AHG}$ & 3 & 0 & 0 \\
\hline 50-ODNG & 3 & 0 & 0 \\
\hline 100-ODNG & 3 & 0 & 0 \\
\hline 200-ODNG & 3 & 0 & 0 \\
\hline Non immunized & 3 & 3 & $4.27 \pm 0.02$ \\
\hline
\end{tabular}

Results are represented as mean $\log \mathrm{CFU} / \mathrm{g} \pm$ standard deviation ( $\mathrm{n}=3$ ). AHG: Aluminium hydroxide group, 50-ODNG: $50 \mu \mathrm{g}$ ODN-DNA group, 100-ODNG: $100 \mu \mathrm{g}$ ODN-DNA group, 200-ODNG: $200 \mu \mathrm{g}$ ODN-DNA group

Table 3. Effect of CpG-ODN adjuvant in broiler chickens immunized with Salmonella Enteritidis bacterin on the intestinal secretory immunoglobulin (SIgA) concentration

\begin{tabular}{|c|c|c|c|c|c|}
\hline \multirow{2}{*}{ Age } & \multirow{2}{*}{$\begin{array}{c}\text { Non Immunized } \\
\text { Control }\end{array}$} & \multicolumn{4}{|c|}{ Dose of CpG-ODN Adjuvant in Salmonella Enteritidis bacterin } \\
\hline & & AHG & 50-ODNG & 100-ODNG & 200-ODNG \\
\hline 18 days & $(5.31 \pm 0.30)$ & $(6.73 \pm 0.06)^{*}$ & $(16.12 \pm 0.25)^{*}$ & $(20.12 \pm 0.29)^{*}$ & $(27.12 \pm 0.29)^{*}$ \\
\hline 35 days & $(3.12 \pm 0.15)$ & $(5.73 \pm 0.25)^{*}$ & $(18.49 \pm 0.40)^{*}$ & $(21.49 \pm 0.42)^{*}$ & $(29.49 \pm 0.22)^{*}$ \\
\hline 42 days & $(1.31 \pm 0.10)$ & $(6.97 \pm 0.058)^{*}$ & $(20.90 \pm 0.08)^{*}$ & $(23.50 \pm 0.28)^{*}$ & $(35.6 \pm 0.13)^{*}$ \\
\hline
\end{tabular}

The results are represented as mean \pm standard deviation $(\mathrm{n}=3)$. * indicate significant differences from non-immunized controls $(\mathrm{P}<0.05)$. AHG: Aluminium hydroxide group, 50-ODNG: $50 \mu \mathrm{g}$ ODN-DNA group, 100-ODNG: $100 \mu \mathrm{g}$ ODN-DNA group, 200-ODNG: 200 $\mu$ g ODN-DNA group.

Table 4. Detection of Salmonella Enteritidis-specific immunoglobulin in serum of broiler chickens immunized Salmonella Enteritidis bacterin adjuvanted with different concentrations of CpG-ODN adjuvant.

\begin{tabular}{lccc}
\hline \multirow{2}{*}{ Group } & \multicolumn{3}{c}{ OD Values } \\
\cline { 2 - 4 } & $\mathbf{1 8}$ days & $\mathbf{3 5}$ days & $\mathbf{4 2}$ days \\
\hline 200-ODNG & 0.657 & 0.867 & 1.0181 \\
100-ODNG & 0.432 & 0.543 & 0.889 \\
50-ODNG & 0.321 & 0.435 & 0.768 \\
AHG & 0.203 & 0.321 & 0.601 \\
NCTG & 0.421 & 0.871 & 1.567 \\
\hline
\end{tabular}

Results are expressed in ELISA optical density values (OD). AHG: Aluminium hydroxide group, 50-ODNG: 50 $\mu$ g ODN-DNA group, 100-ODNG: $100 \mu \mathrm{g}$ ODN-DNA group, 200-ODNG: $200 \mu \mathrm{g}$ ODN-DNA group 

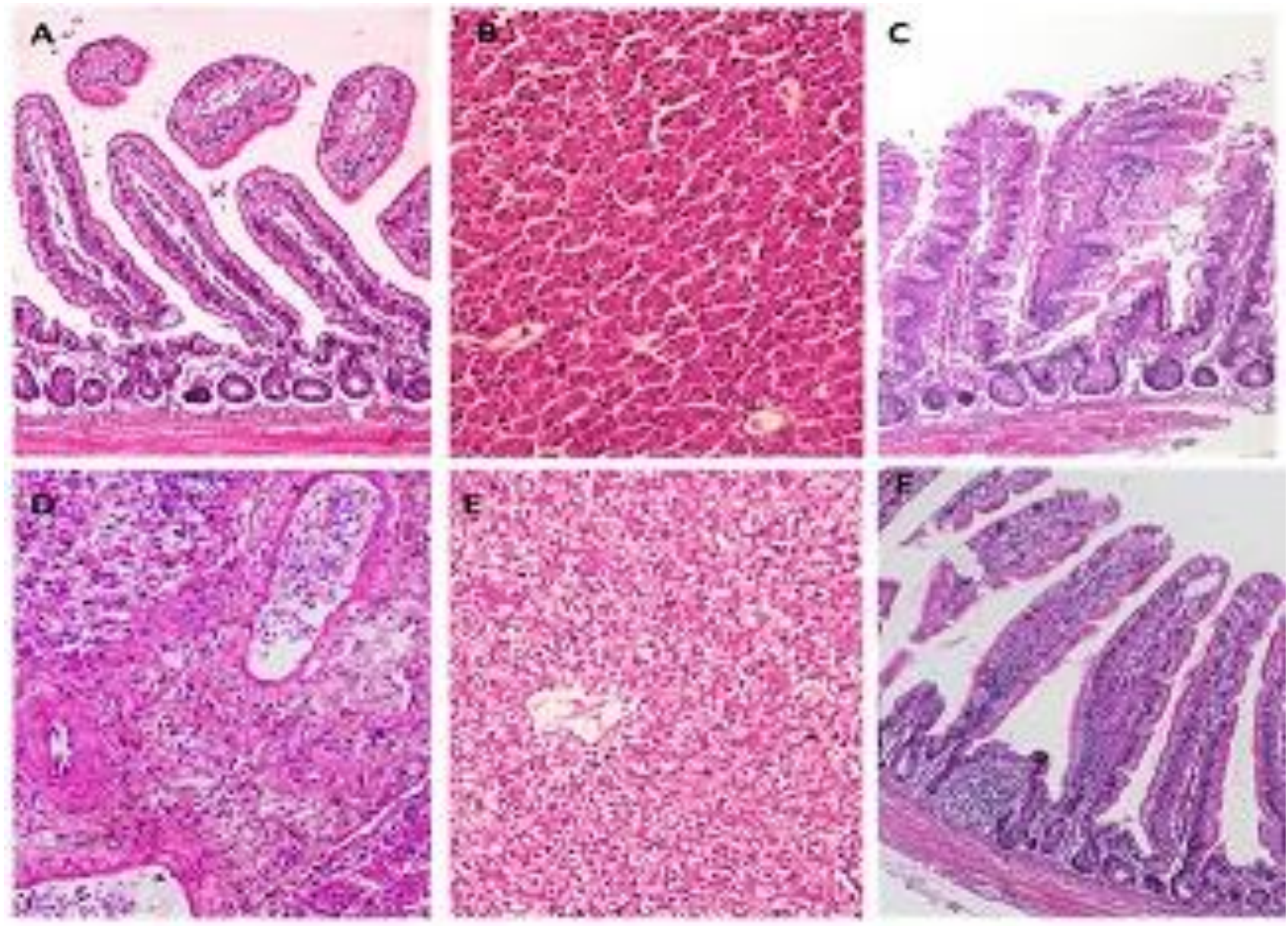

Figure 1. Histopathological images (H\&E staining) from intestinal and liver tissues of broiler chickens at 35 days of age in CpG ODN inoculated and non-inoculated groups. A: Intestine of chicken in 200-ODNG group showing apparently normal histological structure (X100). B: Liver of chicken in 200-ODNG group showing apparently normal histological structure (X200).C: Intestine of chicken in non-vaccinated group (NVG) showing severe epithelial and goblet cells hyperplasia (X100). D: Liver of chicken in N NVG showing increased portal fibrous connective tissue with few mononuclear cells infiltration and vacuolation of adjacent hepatocytes (X200). E: Liver of chicken in chickens immunized showing mild to moderate vacuolated hepatocytes (X200). F: Intestine of chicken in AHG adjuvant group showing focal mononuclear cells infiltration and slight goblet cell hyperplasia (X100).

\section{DISCUSSION}

The current study provides further insights on the interaction of Salmonella with avian host and highlights the role CpGODN in immunity against these leading food-borne zoonotic bacteria. In this study, the CpG-ODN adjuvanted Salmonella Enteritidis bacterin had significantly improved the survival rate of Salmonella Enteritidis-infected chickens. Moreover and unlike the negative control group, chicken groups immunized with CpG-ODN adjuvanted Salmonella Enteritidis bacterin were able to clear Salmonella Enteritidis colonization from the intestine. In this study, the lack of Salmonella recovery from the intestinal tract of challenged immunized chickens could be reasoned to the bactericidal activity of CpG DNA. Indeed, previous literature have shown that CpG-DNA possess an antimicrobial activity inducing microbial killing by stimulation of nitric oxide and oxygen production from defense cells that limit the systemic spread of Salmonella (Xie et al., 2003; He et al., 2007).

Data from this study have shown a dose dependent effect of CpG ODN on induction of SIgA. The CpG ODN appeared to have strong immune stimulatory activity on SIgA response in all tested time points with the 200-ODNG group showed the highest response followed by 100-ODNG group then the 50-ODNG and the AHG groups $(\mathrm{P}<0.05)$. This finding is comparable with previously published researches, which strongly support that CpG ODN stimulates host defense mechanisms against pathogens, involving both innate and acquired immune responses (Krieg, 2002). The obtained results have shown that CpG-ODN induces a significant increase in the mucosal immune responses, which could play a major role in containing Salmonella infection in the gut, via Th2 allergic pathway rather than Th1 inflammatory immune responses. These results could be influenced by the Salmonella strain, age and dose of challenge, age of sampling or concentration of CpG-ODN.

In the present study, cellular responses were recorded in intestinal and liver tissues at 35 days of age using $\mathrm{H} \& \mathrm{E}$ histopathological staining. Contrary to serological results, tissue samples from AHG have shown mild to moderate tissue reaction while liver and intestinal tissue samples taken from chickens in the 200-ODNG group showed no apparent cellular changes. These findings contradict previous researches which demonstrate, in vitro, the immune-modulatory 
activity of $\mathrm{CpG}$ ODN through modulation of neutrophil apoptosis and reactive oxygen production with further activation inflammatory responses via IL-1B and nitric oxide production in stimulated avian macrophages (Sanjaya et al., 2017; Golenkina et al., 2019). This could be reasoned by other contributing factors, including the age of sampling and Salmonella strain used. Indeed, Salmonella Enteritidis infection upregulates mRNA gene expression of proinflammtory cytokines and chemokines, including CXCLi1, CXCLi2, iNOS and IL-6 in the caecal tonsils and HD11 macrophages (Setta et al., 2012a and 2012b). However, data from this paper has shown that the administration of CpG ODN motifs were able to enhance chicken survival and inhibit intestinal colonization following oral exposure to Salmonella Enteritidis, with stimulation of mucosal SIgA in a dose-dependent manor. Thus, it appeared that the CpG ODN-induced local immune response has played an important role in the mucosal responses, again pointing out the possible role for Th2 responses. Further studies might be needed to investigate the allergic immune responses to CpG ODN in vivo in chicken model.

\section{CONCLUSION}

This study stating an interesting data since Salmonella continues to present a major threat to poultry industry as well as of public health burden with poultry as the main source of infection. The present paper provides an update about the mucosal and cellular responses of chickens to Salmonella Enteritidis following administration of Toll-like receptor (TLR)-21-agonist oligodeoxynucleotide containing Cytosine Guanine motifs CpG DNA. In response to CpG DNA administration, chickens were able to clear Salmonella from enteric sites. This research study also shows that CpG DNA modulates immune responses to Salmonella as it induces significant immune stimulatory activity demonstrated by increased secretory IgA production and remarked reduction in cellular interactions. Further studies on the gene expression of immune markers representing Th1/Th2 paradigm in response to CpG ODN are perhaps needed.

\section{DECLARATIONS}

\section{Acknowledgments}

The authors would like to acknowledge Ceva Santé Animale, Egypt for funding this study.

\section{Competing interests}

The authors declare that they have no competing interests.

\section{Author's contributions}

All authors participated in making the design, support with sampling, interpretation of results and writing the paper. Mohamed Abed and Mahmoud Elhariri did the experiments, Ahmed Setta, Mahmoud Elhariri and Rafik Soliman designed the experiment and analysed the results. Mohamed Abed, Mahmoud Elhariri, Rehab El-Helw, Marwa S. Khattab, Ahmed Setta and Rafik Soliman wrote and revised the manuscript.

\section{Competing interests}

The authors declare that there is no conflict of interest.

\section{REFERENCES}

Barrow PA (2007). Salmonella infections: immune and non-immune protection with vaccines. Avian Pathology, 36: 1-13. DOI: https://10.1080/03079450601113167

Burr R, Effler P, Kanenaka R, Nakata M, Holland B and Angulo FJ (2005). Emergence of Salmonella serotype Enteritidis phage type 4 in Hawaii traced to locally-produced eggs. International Journal of Infectious Diseases, 9: 340-346. DOI: https://10.1016/2004.10.004

Ciraci C and Lamont SJ (2011). Avian-specific TLRs and downstream effector responses to CpG-induction in chicken macrophages. Developmental and Comparative Immunology, 35: 392-398. DOI: https://10.1016/2010.11.012

Golenkina EA, Viryasova GM, Galkina SI, Arifulin EA, Gaponova TV, Romanova YM and Sud'ina GF (2019). Synthetic CpG oligonucleotides as potential modulators of neutrophil survival in PAMP-associated inhibition of apoptosis. Journal Leukocyte Biolology, 106(1):45-55. DOI: https://10.1002/1118-435r

Haiqi H, Genovese KJ, Swaggerty, CL, Nisbet, DJ and Kogut and MH (2008). Differential induction of nitric oxide, degranulation, and oxidative burst activities in response to microbial agonist stimulations in monocytes and heterophils from young commercial turkeys. Veterinary Immunology and Immunopathology, 123:177-185. DOI: https://10.1016/.2008.01.033

He H, Genovese KJ, Swaggerty CL, Nisbet DJ and Kogut MH (2007). In vivo priming heterophil innate immune functions and increasing resistance to infection in neonatal chickens by immune stimulatory $\mathrm{CpG}$ oligodeoxynucleotides. Veterinary Immunology and Immunopathology, 117: 275-283. DOI: https://10.1016/2007.03.002

Janeway CA, JR and Medzhitov R (2002). Innate immune recognition. Annual Reviews of Immunology, 20: 197-216. DOI: https://10.1146/.20.083001.084359 
Keestra AM, de Zoete MR, Bouwman, LI and van Putten JP (2010). Chicken TLR21 is an innate CpG DNA receptor distinct from mammalian TLR9. Journal of Immunology, 185: 460-467. DOI: 10.4049/.0901921

Krieg AM (2002). CpG motifs in bacterial DNA and their immune effects. Annual Reviews of Immunolology, 20: 709-60. DOI: https://10.1146/.20.100301.064842

Much P, Pichler J and Allerberger F (2007). Food borne infectious outbreaks, Austria 2005. Wien Klin Wochenschr, 119: 150-157. DOI: https://10.4049/.0901921

Ribeiro CM and Schijns VE (2010). Immunology of vaccine adjuvants. Methods in Molecular Biology, 626: 114. DOI: https://10.1007/978-1-60761-585-91

Sanjaya A, Elder JR and Shah DH (2017). Identification of new CpG oligodeoxynucleotide motifs that induce expression of interleukin- $1 \beta$ and nitric oxide in avian macrophages. Veterinary Immunolology Immunopathology, 192:1-7. DOI: https://10.1016/.2017.08.005

Setta A, Barrow PA, Kaiser P and Jones MA (2012a). Early immune dynamics following infection with Salmonella enterica serovars Enteritidis, Infantis, Pullorum and Gallinarum; cytokine and chemokine gene expression profile and cellular changes of chicken cecal tonsils. Comparative Immunology, Microbiology and Infectious Diseases, 35(5): 397-410. DOI: https://doi.org/10.1016/j.cimid.2012.03.004

Setta A, Barrow PA, Kaiser P and Jones MA (2012b). Immune dynamics following infection of avian macrophages and epithelial cells with typhoidal and non-typhoidal Salmonella enterica serovars; bacterial invasion and persistence, nitric oxide and oxygen production, differential host gene expression, NF- $\mathrm{BB}$ signalling and cell cytotoxicity. Veterinary Immunology and Immunopathology, 146(3-4):212-224. DOI: 10.1016/j.vetimm.2012.03.008

Stephens N, Sault C, firestone SM, Lightfoot D and Bell C (2007). Large outbreaks of Salmonella Typhimurium phage type 135 infections associated with the consumption of products containing raw egg in Tasmania. Communicable Disease Intelligence, 31: 118-124. PMID: 17503652

Tovey MG and Lallemand C (2010). Adjuvant activity of cytokines. Methods in Molecular Biology, 626: 287-309. DOI: https://10.1007/978-1-60761-585-919

Xie H, Raybourne RB, Babu US, Lillehoj HS and Heckert RA (2003). CpG-induced immunomodulation and intracellular bacterial killing in a chicken macrophage cell line. Developmental and Comparative Immunology, 27: 823-834. DOI: https://10.1016/s0145-305x(03)00079-x 